10,09

\title{
Расчеты ab initio дисперсии фононов в $\mathrm{CdGa}_{2} \mathrm{~S}_{4}$
}

\author{
(C) З.А. Джахангирли ${ }^{1,2}$, Т.Г. Керимова ${ }^{1 . \mp, ~ И . А . ~ М а м е д о в а ~}{ }^{1}$, Н.А. Абдуллаев ${ }^{1}$, Н.Т. Мамедов ${ }^{1}$ \\ ${ }^{1}$ Институт ффизики им. Г.М. Абдуллаева НАН Азербайджана, \\ Баку, Азербайджан \\ ${ }^{2}$ Азербайджанский технический университет, \\ Баку, Азербайджан \\ ฯ E-mail: taira-kerimova@mail.ru, zakircahangirli@yahoo.com
}

(Поступила в Редакцию 23 ноября 2017 г.

В окончательной редакции 9 марта 2018 г.)

Исследован фононный спектр $\mathrm{CdGa}_{2} \mathrm{~S}_{4}$ экспериментально с использованием рамановской спектроскопии, и теоретически, используя теорию функционала плотности. Регистрированы и идентифицированы одиннадцать активных комбинационных мод в $83,135,165,219,243,260,310,322,352,361,392 \mathrm{~cm}^{-1}$. Идентификация всех колебательных мод была получена из рассмотрения точечной группы симметрии. Проведено также сравнение с результатами имеющихся в литературе экспериментальными данными, полученными методами инфракрасной спектроскопии и комбинационного рассеяния света.

Настоящая работа выполнена при финансовой поддержке Фонда развития науки при президенте Азербайджанской Республики (грант № EIF-KETPL-2-2015-1(25)-56/02/1).

DOI: 10.21883/FTT.2018.11.46675.338

\section{1. Введение}

Соединения группы $A^{\mathrm{II}} B_{2}^{\mathrm{III}} C_{4}^{\mathrm{VI}}$ кристаллизующиеся в пространственной группе $S_{4}^{2}$, являясь кристаллохимическими аналогами соединений, кристаллизующихся в структуре сфалерита и халькопирита, привлекают внимание исследователей, в связи с перспективностью применения их в оптоэлектронике и в полупроводниковом приборостроении [1,2]. Для этих соединений характерны оптическая анизотропия, двулучепреломление, значительные величины коэффициентов нелинейной восприимчивости, высокая фоточувствительность, яркая люминесценция. Поэтому исследование физических свойств этих соединений является важной задачей. Особое место занимают исследования электронных и фононных состояний как экспериментально, так и теоретически. Знание фононных спектров, их зависимость от температуры, давления и других внешних факторов важны при объяснении механизмов теплоемкости, теплового расширения, теплопроводности, поглощения звука и др. Совместные теоретические и экспериментальные исследования фононных спектров, динамики решетки позволяют получить информацию о частотах фононов в центре зоны Бриллюэна (ЗБ) и электрон-фононном, фонон-фононном взаимодействиях и др.

Симметрия фононных состояний впервые методом теории групп исследована в [3]. В работе [4]исследованы спектры инфракрасного (ИК) поглощения и комбинационного рассеяния света (КРС) в неполяризованном излучении порошкообразного $\mathrm{CdGa}_{2} \mathrm{~S}_{4}$. Ранее нами также исследованы инфракрасные спектры отражения и спектры комбинационного рассеяния света в $\mathrm{CdGa}_{2} \mathrm{~S}_{4}[5,6]$. В результате совместного анализа поляризационных зависимостей оптических фононов (КРС и ИК) и симметризованных смещений атомов в эле- ментарной ячейке были идентифицированы оптические фононы по типам симметрии, определены силы осцилляторов, полуширины линий и др. параметры. В [7-9] проведены экспериментальные исследования спектров ИК отражения и КРС ряда соединений $A^{\mathrm{II}} B_{2}^{\mathrm{III}} C_{4}^{\mathrm{VI}}$, в том числе, $\mathrm{CdGa}_{2} \mathrm{~S}_{4}$ и твердых растворов на их основе. $\mathrm{B}$ [10-13] экспериментально и теоретически исследовано влияние давления на фононный спектр в $\mathrm{CdGa}_{2} \mathrm{~S}_{4}$.

В настоящей работе с целью получения дополнительной информации о динамических свойствах $\mathrm{CdGa}_{2} \mathrm{~S}_{4}$, были проведены расчеты дисперсии и плотности фононных состояний $a b$ initio, основанных на формализме теории функционала плотности. Электронный спектр и оптические функции этого соединения экспериментально и теоретически исследованы достаточно полно, в то время как динамические характеристики, определяющие термодинамические характеристикии оптические свойства кристаллов в ИК области исследованы недостаточно. Поэтому, являются актуальным как теоретические, так и экспериментальные исследования динамики решетки данного соединения. Теоретический расчет динамической структуры позволил нам идентифицировать симметрию рамановских мод и обсудить экспериментальные результаты. Одновременно были проведены исследования спектров КРС на конфокальном рамановском микроспектрометре.

\section{2. Методика эксперимента, кристаллическая структура и метод расчета}

Монокристаллы $\mathrm{CdGa}_{2} \mathrm{~S}_{4}$ были выращены методом газотранспортных реакций. В качестве транспортера использовался кристаллический йод. Параметры, опреде- 
Таблица 1. Оптимизированные и экспериментальные параметры решетки $\mathrm{CdGa}_{2} \mathrm{~S}_{4}$

\begin{tabular}{c|c|c}
\hline Параметры & Теория & Эксперимент $[14]$ \\
\hline$a, \AA$ & 5.4272 & 5.55 \\
$c, \AA$ & 9.9297 & 10.19 \\
$c / a$ & 1.8296 & 1.834
\end{tabular}

ленные рентгенографически, кристаллической решетки, пространственная группа $S_{4}^{2} \mathrm{CdGa}_{2} \mathrm{~S}_{4}$ [14] приведены в табл. 1.

Измерения спектров комбинационного рассеяния в $\mathrm{CdGa}_{2} \mathrm{~S}_{4}$ были проведены на конфокальном рамановском микроспектрометре Nanofinder 30 (Tokyo Instr., Japan). В качестве источника возбуждения использовался лазер $\mathrm{Nd}$ :YAG с выходной длиной волны излучения $\lambda=532 \mathrm{~nm}$ и максимальной мощностью $10 \mathrm{~mW}$. Спектральное разрешение было не хуже $0.5 \mathrm{~cm}^{-1}$. Детектором рассеянного излучения являлась охлаждаемая (термоэлектрическим способом до $-100^{\circ} \mathrm{C}$ ) $\mathrm{CCD}$ камера (1024 на 128 пикселя), работающая в режиме счета фотонов. Измерения спектров КРС проводились в геометрии обратного рассеяния в неполяризованном свете.

Все вычисления были выполнены $a b$ initio, ocнованных на DFPT (Density Functional Perturbation Theory) [15-17] с использованием метода псевдопотенциала на основе плоских волн, реализованного в коде ABINIT [18]. В этой работе были использованы сохраняющие нормы псевдопотенциалы Hartwigsen-GoedeckerHutter [19]. Обменно-корреляционное взаимодействие описывалось в обобщенном градиентном приближении (GGA), как указано в [20]. В разложении волновых функций были учтены плоские волны с энергией до $80 \mathrm{Ry}$, что обеспечивает хорошую сходимость полной энергии. Суммирования по зоне Бриллюэна выполнялись на сетке $4 \times 4 \times 4$ Монкхорста-Пака [21]. Параметры решетки и равновесные положения атомов в элементарной ячейке определялись минимизацией сил Геллмана-Фейнмана. Равновесные положения атомов в элементарной ячейке были найдены методом BFGS (Broyden-FletcherGoldfarb-Shanno) с использованием экспериментальных данных в качестве начальных значений. Процесс минимизации продолжался до тех пор, пока силовые модули не оказывались меньше $10^{-8} \mathrm{Ry} / \mathrm{Bohr}$. При расчете плотности фононных состояний была использована сетка $40 \times 40 \times 40$ точек в ЗБ. После оптимизации кристаллической структуры были рассчитаны фононный спектр и плотность фононных состояний $\mathrm{CdGa}_{2} \mathrm{~S}_{4}$. Динамика решетки определялась с использованием DFPT, где статический линейный отклик на фононные искажения определяется электронными свойствами основного состояния. DFPT позволяет получить динамические матрицы для любой точки ЗБ.

Вначале была проверена зависимость сходимости полной энергии и сил Геллмана-Фейнмана от сетки
Монхорста-Пака и от максимальной энергии плоских волн с учетом оптимального употребляемого машинного времени для вычислений. Расчеты показали, что сетка $4 \times 4 \times 4$ и максимальная энергия плоских волн $80 \mathrm{Ry}$ в разложении волновых функций дают достаточно хорошие результаты для спектров комбинационного рассеяния и других динамических свойств $\mathrm{CdGa}_{2} \mathrm{~S}_{4}$.

\section{3. Обсуждение полученных результатов}

Элементарная ячейка содержит 7 атомов и соответственно, фононный спектр состоит из 21 решеточной моды. Нормальные моды в центре ЗБ $\mathrm{CdGa}_{2} \mathrm{~S}_{4}$ описываются неприводимыми представлениями пространственной группы $S_{4}^{2}$,

$$
\Gamma=3 A(\mathrm{RS})+6 B(\mathrm{IR}, \mathrm{RS})+6 E(\mathrm{IS}, \mathrm{RS}) .
$$

Нормальные моды $A, B$ и $E$ симметрии активны в комбинационном рассеянии (RS). Поскольку, в $\mathrm{CdGa}_{2} \mathrm{~S}_{4}$ отсутствует центр инверсии, снимается правило альтернативного запрета, в КР могут проявляться ИК-активные моды. Моды $B$ и $E$ симметрии активны также в спектрах инфракрасного поглощения (IR) в поляризации $E \| c$ и $E \perp c$, соответственно. Одна мода симметрии $B$ и одна двукратно вырожденная мода $E$ являются акустическими.

На рис. 1 представлен спектр КРС в $\mathrm{CdGa}_{2} \mathrm{~S}_{4}$. Как видно, в спектре наблюдаются 11 мод, значения которых приведены в табл. 2.

Из анализа вектора смещения атомов для моды $A$ симметрии можно сделать вывод, что полносимметричные колебания типа $A$ связаны со смещениями атомов анионной подрешетки вдоль кристаллографических осей $x, y, z$. В колебаниях $B$ симметрии атомы катионной подрешетки смещаются относительно анионной подрешетки вдоль тетрагональной оси $c$. Колебания симметрии $E_{x}$ и $E_{y}$ связаны со смещениями атомов катионной подрешетки вдоль кристаллографических осей $x$ и $y$, соответственно.



Рис. 1. Спектр KРC $\mathrm{CdGa}_{2} \mathrm{~S}_{4}$. 
Таблица 2. Экспериментально определенные и вычисленные с учетом макроскопического электрического поля в направлении волнового вектора [100] (в декартовых координатах) частоты оптических фононов в $\mathrm{CdGa}_{2} \mathrm{~S}_{4}$

\begin{tabular}{|c|c|c|c|c|c|}
\hline \multirow[b]{2}{*}{ Симметрия } & \multirow{2}{*}{$\begin{array}{c}\omega_{\text {theo }}, \mathrm{cm}^{-1} \\
\text { наст. раб. }\end{array}$} & \multicolumn{4}{|c|}{$\omega_{\text {exp }}, \mathrm{cm}^{-1}$} \\
\hline & & $\begin{array}{c}\text { IR [12] } \\
\text { (TO/LO) }\end{array}$ & $\begin{array}{c}\text { RS [6] } \\
\text { (TO/LO) }\end{array}$ & $\begin{array}{c}\text { RS [7] } \\
\text { (TO/LO) }\end{array}$ & $\begin{array}{c}\text { RS дан. } \\
\text { раб. (TO/LO) }\end{array}$ \\
\hline $\begin{array}{l}A_{1} \\
A_{2} \\
A_{3}\end{array}$ & $\begin{array}{l}221.67 \\
311.54 \\
352.88\end{array}$ & & $\begin{array}{l}220 \\
313.5 \\
367\end{array}$ & $\begin{array}{l}218 \\
308 \\
359\end{array}$ & $\begin{array}{l}219 \\
310 \\
352\end{array}$ \\
\hline $\begin{array}{l}B_{1} \\
B_{2} \\
B_{3} \\
B_{4} \\
B_{5}\end{array}$ & $\begin{array}{r}87.85 \\
165.66 \\
260.62 \\
316.54 \\
369.01\end{array}$ & $\begin{array}{l}162 / 168 \\
238 / 245 \\
262 / 269 \\
330 / 343 \\
367 / 393\end{array}$ & $\begin{array}{l}166 / 170 \\
242 / 244 \\
256 / 266 \\
326 / 342 \\
364 / 394\end{array}$ & $\begin{array}{c}- \\
165 \\
257 \\
291 \\
371 / 389\end{array}$ & $\begin{array}{c}165 \\
- \\
260 \\
322 \\
361 / 392\end{array}$ \\
\hline $\begin{array}{l}E_{1} \mathrm{LO} \\
E_{2} \mathrm{LO} \\
E_{3} \mathrm{LO} \\
E_{4} \mathrm{LO} \\
E_{5} \mathrm{LO}\end{array}$ & $\begin{array}{c}88.75 \\
138.03 \\
253.48 \\
349.77 \\
377.64\end{array}$ & $\begin{array}{c}- \\
144 \\
253 \\
346 \\
385\end{array}$ & $\begin{array}{r}88 \\
136 \\
244 \\
348 \\
392\end{array}$ & $\begin{array}{r}86 \\
136 \\
237 \\
320 \\
382\end{array}$ & $\begin{array}{r}83 \\
135 \\
243\end{array}$ \\
\hline $\begin{array}{l}E_{1} \mathrm{TO} \\
E_{2} \mathrm{TO} \\
E_{3} \mathrm{TO} \\
E_{4} \mathrm{TO} \\
E_{5} \mathrm{TO}\end{array}$ & $\begin{array}{c}85.78 \\
137.33 \\
249.36 \\
321.92 \\
355.57\end{array}$ & $\begin{array}{r}86 \\
136 \\
238 \\
318 \\
360\end{array}$ & $\begin{array}{r}82 \\
120 \\
240 \\
332 \\
364\end{array}$ & 365 & \\
\hline
\end{tabular}

На рис. 2 представлен рассчитанный фононный спектр вдоль основных направлений ЗБ для $\mathrm{CdGa}_{2} \mathrm{~S}_{4}$. Как видно из рисунка, дисперсия оптических фононов в направлении $\Gamma-Z$ вдоль тетрагональной оси $c$ незначительна, что указывает на слабое фонон-фононное взаимодействие. Дисперсия оптических фононов в направлении $\Gamma-X-P-N$ значительна, что указывает на сильное межатомное взаимодействие. Кроме того, фононные спектры обнаруживают анизотропию во всех направлениях в ЗБ за счет ковалентной связи $\mathrm{Cd}-\mathrm{S}$ и $\mathrm{Ga}-\mathrm{S}$. Из расчетов максимальная частота фононов получается равной $390 \mathrm{~cm}^{-1}$. Динамический дипольный момент, связанный с инфракрасно активными продольными оптическими $B$ и $E$ модами, вызывает LO-TO

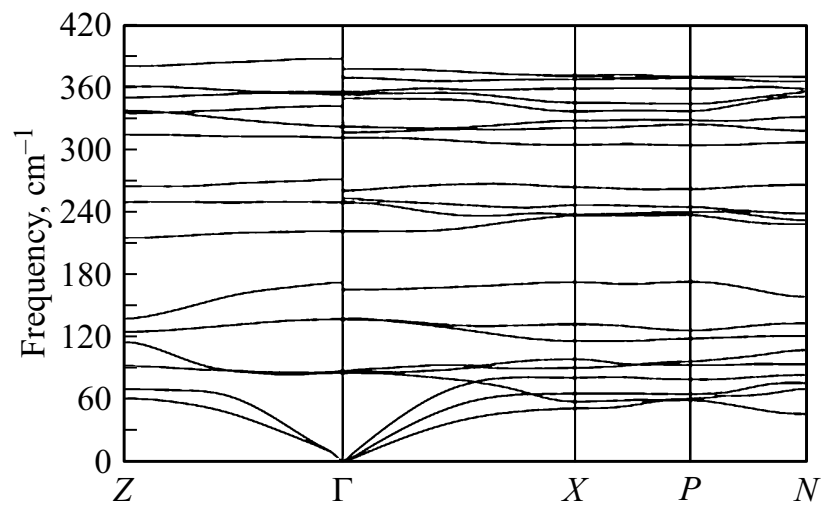

Pис. 2. Дисперсия фононов в $\mathrm{CdGa}_{2} \mathrm{~S}_{4}$. расщепление. Эти расщепления в центре ЗБ появляются как разрывы в фононных спектрах, которые хорошо видны на рис. 2. Основные максимумы фононной плотнотности состояний находятся в $75,82,94,131,167,246$, $266,349,359$ и $365 \mathrm{~cm}^{-1}$. Кроме того, фононный DOS охватывает диапазон частот от 0 до $380 \mathrm{~cm}^{-1}$ и состоит из трех областей, разделенных широким энергетическим зазором от 175 до $215 \mathrm{~cm}^{-1}$ и от 271 до $303 \mathrm{~cm}^{-1}$. Эти широкие энергетические щели являются результатом большой разности масс между атомами $\mathrm{Cd}, \mathrm{Ga}$ и $\mathrm{S}$, что приводит к расщеплению катионных и анионных колебаний.

На рис. 3 приведены проектированные на атомы плотности фононных состояний (DOS) $\mathrm{CdGa}_{2} \mathrm{~S}_{4}$. Анализ проектированного на атомы фононный DOS показывает, что в первой области от 0 до $83 \mathrm{~cm}^{-1}$ с максимумом при $82 \mathrm{~cm}^{-1}$ преобладают движения $\mathrm{Cd}$ и включают акустические и низкочастотные оптические ветви с В и Е модами, в то время как верхняя часть первой области от 83 до $175 \mathrm{~cm}^{-1}$ с основным максимумом при $94 \mathrm{~cm}^{-1}$ является следствием связанных движений $\mathrm{Ga}$ и $\mathrm{S}$ в элементарной ячейке. Вторая область обусловлена смещением атомов $\mathrm{S}$, но с небольшим вкладом атомов $\mathrm{Ga}$ и $\mathrm{Cd}$. Высокочастотная третья область, в основном, связана с движением атомов серы, что сопровождается изменениями длины валентной связи. Можно считать, что в этой области атомы $\mathrm{Cd}$ остаются на своих местах и не участвуют в колебаниях. Фононные моды $A$ симметрии являются высокочастотными и начинаются с $220 \mathrm{~cm}^{-1}$. 


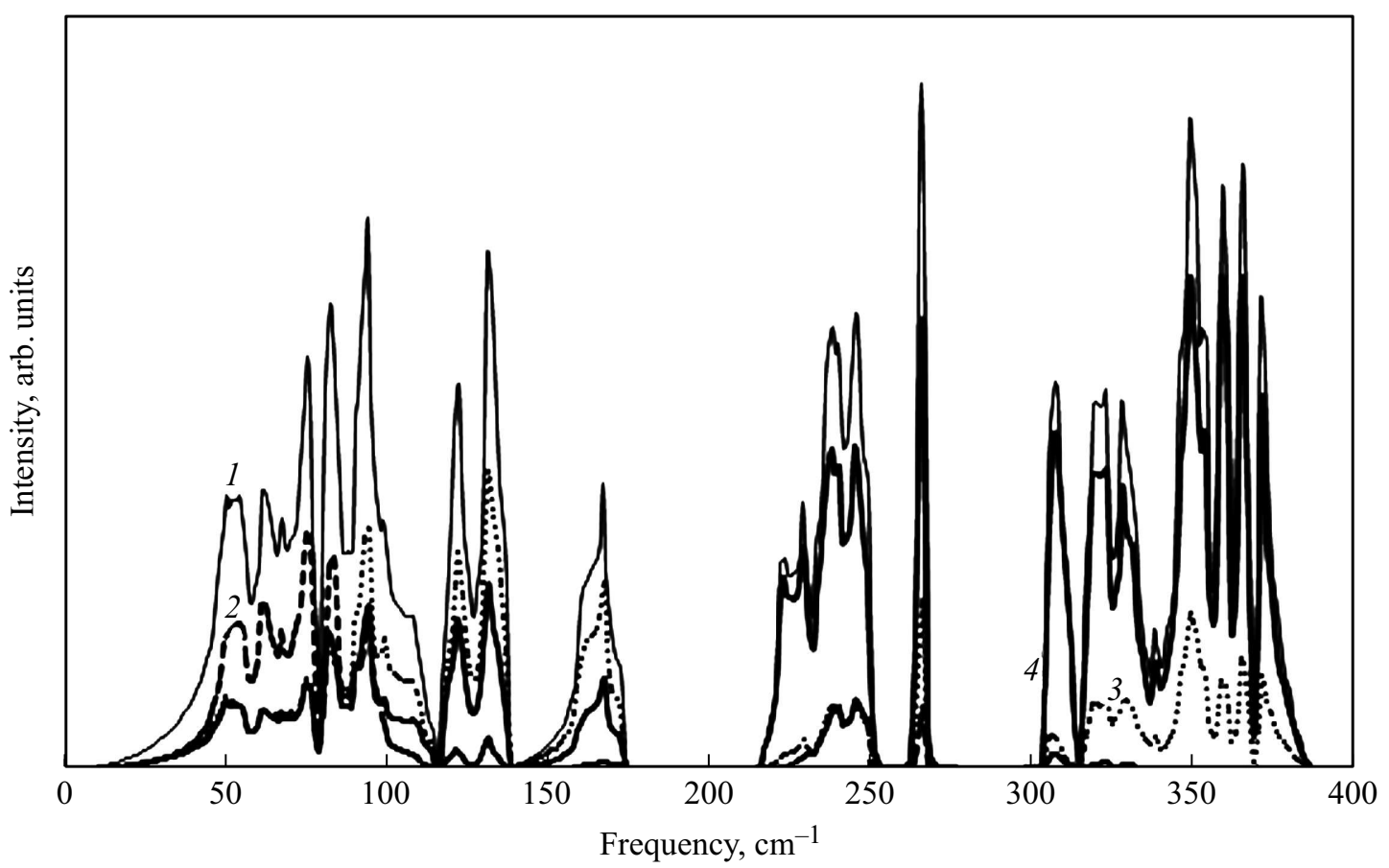

Рис. 3. Проектированный на атомы фононный $\mathrm{DOS}$ в $\mathrm{CdGa}_{2} \mathrm{~S}_{4}: 1$ - полный фононный DOS, 2 - проектированный на атомы Cd фононный DOS, 3 - проектированный на атомы Ga фононный DOS, 4 - проектированный на атомы S фононный DOS.

В табл. 2 представлены рассчитанные при оптимизированных параметрах решетки с учетом макроскопического электрического поля в направлении волнового вектора [100] (в декартовых координатах) фононные частоты вместе с результатами, полученными из ИКи КР-спектров $\mathrm{CdGa}_{2} \mathrm{~S}_{4}$. Как видно из таблицы, теоретические и экспериментальные частоты находятся в удовлетворительном согласии.

\section{4. Теплоемкость}

Зная плотность фононных состояний $g(\omega)$, можно вычислить свободную энергию $F(T)$

$$
F(T)=-\int_{0}^{\omega_{m}}\left\{\frac{\hbar \omega}{2}+k_{\mathrm{B}} T \ln \left[2 n_{B}(\omega)\right]\right\} g(\omega) d \omega,
$$

здесь фактор Бозе-Эйнштейна $n_{B}\left(\omega_{q j}\right)=\left[e^{\hbar \omega_{q j} / k_{\mathrm{B}} T}-1\right]$, $k_{\mathrm{B}}$ - постоянная Больцмана, $\omega_{m}$ - наивысшая частота в $g(\omega)$, определяющая верхний предел интегрирования, а также теплоемкость при постоянном объеме $C_{V}(T)$

$$
C_{V}(T)=-T\left(\frac{\partial^{2} F}{\partial T^{2}}\right)_{V} .
$$

На рис. 4 приведены теоретически рассчитанные величины теплоемкости при постоянном объеме $C_{V}$ вплоть до $400 \mathrm{~K}$ (сплошная линия) и экспериментально измеренные авторами [22] до $300 \mathrm{~K}$ (черные точки) значения теплоемкости при постоянном давлении $C_{p}(T)$ для $\mathrm{CdGa}_{2} \mathrm{~S}_{4}$. Как видно из рис. 4 , в величинах $C_{p}$ и $C_{V}$ наблюдается некоторое малое различие.
Как известно, разница $C_{p}(T)-C_{V}(T)$ определяется фундаментальным термодинамическим соотношением

$$
C_{p}-C_{V}=T\left(\frac{\partial p}{\partial T}\right)_{V}\left(\frac{\partial V}{\partial T}\right)_{p}=-\left(\frac{\partial p}{\partial V}\right)_{T}\left(\frac{\partial V}{\partial T}\right)_{p}^{2} .
$$

Соотношение (4) можно записать как

$$
C_{p}-C_{V}=T V \frac{\alpha^{2}}{\beta}
$$

здесь $T$ - температура, $V$ - молярный объем, $\alpha-$ коэффициент теплового расширения, $\beta$ - изотермическая сжимаемость.

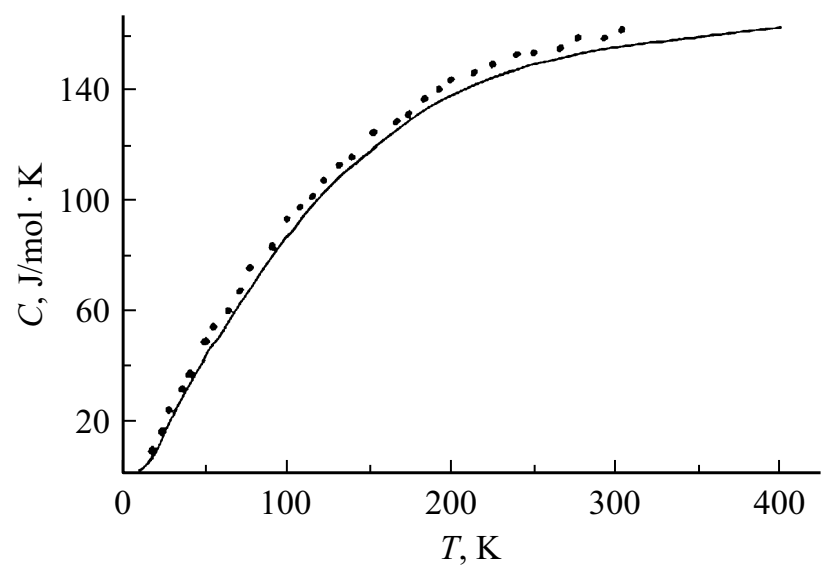

Рис. 4. Температурная зависимость теплоемкости $\mathrm{CdGa}_{2} \mathrm{~S}_{4}$ (сплошная линия - расчет, точки - эксперимент [22]). 
Учитывая, что молярный объем $V=M / \rho$, где молярная масса $M \approx 380 \mathrm{~g} / \mathrm{mol}$, а плотность $\rho=4.03 \mathrm{~g} / \mathrm{cm}^{3}$ [23], находим $V \approx 94.3 \mathrm{~cm}^{3} / \mathrm{mol}$. Из [24] коэффициент теплового расширения для $\mathrm{CdGa}_{2} \mathrm{~S}_{4} \quad \alpha=2 \alpha_{a}+\alpha_{c}$ $\approx 23.5 \cdot 10^{-6} \mathrm{~K}^{-1}$, а из $[25]$ изотермическая сжимаемость $1 / \beta=B \approx 58.4 \mathrm{GPa}$, где $B-$ изотермический объемный модуль упругости.

Таким образом, оценки согласно (5) дают значение $C_{p}(300 \mathrm{~K})-C_{V}(300 \mathrm{~K}) \sim 0.91 \mathrm{~J} / \mathrm{mol} \cdot \mathrm{K}$, что согласуется с данными представленными на рис. 4. Малая величина разности $C_{p}(T)-C_{V}(T)$ свидетельствует о слабом ангармонизме сил взаимодействия атомов внутри кристаллической решетки.

\section{5. Заключение}

На основе расчетов ab initio в рамках теории функционала плотности исследована дисперсия и плотность фононных состояний монокристаллов $\mathrm{CdGa}_{2} \mathrm{~S}_{4}$. Анализ, проектированный на атомы, плотности фононных состояний показывает, что плотность состояний $\mathrm{CdGa}_{2} \mathrm{~S}_{4}$ состоит из трех областей. В первой части, охватывающая область от 0 до $175 \mathrm{~cm}^{-1}$, в основном, участвуют низкочастотные оптические и акустические фононы и частично атомы анионов. Вторая область, от 175 до $215 \mathrm{~cm}^{-1}$ обусловлена смещением атомов $\mathrm{S}$ и с небольшим вкладом $\mathrm{Ga}, \mathrm{Cd}$. Третья, высокочастотная область, от 271 до $380 \mathrm{~cm}^{-1}$, в основном, связана с движением атомов $\mathrm{S}$, что сопровождается с изменением длины валентной связи.

Также исследована температурная зависимость теплоемкости в интервале $5-400 \mathrm{~K}$. Незначительное различие в значениях $C_{p}(T)$ и $C_{V}(T)$ свидетельствует о слабом ангармонизме сил взаимодействия атомов внутри кристаллической решетки.

Авторы считают своим долгом выразить благодарность А. Бондякову (ОИЯИ, Дубна, Россия), а также всему коллективу дата-центра AZGRID, Институту Физики НАН Азербайджана, за предоставленные ресурсы и техническую поддержку теоретических расчетов.

\section{Список литературы}

[1] A.N. Georgobiani, S.I. Radautsan, I.M. Tiginyanu. Semiconductors 19, 193 (1985).

[2] I.S. Yahia, M. Fadel, G.B. Sakr, F. YakubHanoglu, S.S. Shenouda, W.A. Faroog. J. Alloys Compd. 509, 4414 (2011).

[3] Т.Г. Керимова. Докл. АН Азерб. ССР, XXXV, 30 (1979).

[4] В.Ю. Сливка, Е.Ю. Переш, Л.М. Сусликов, В.С. Герасименко, М.Ю. Риган. УФЖ 22, 1951(1977).

[5] Т.Г. Керимова, Р.Х. Нани, Э.Ю. Салаев, В.Я. Штейншрайвер. ФТТ 21, 2791 (1979).

[6] Т.Г. Керимова, А.Ш. Хидыров, Э.Ю. Салаев, В.Я. Штейншрайвер. ФТТ 27, 1570 (1985).

[7] P.P. Lottici, C. Razzetti. IL Nuovo Cimento 2 D 6, 2050 (1983).

[8] C. Razzetti, P.P. Lottici, L. Zanotti, M. Curti. Phys. Status Solidi B 118, 743 (1983).
[9] P.P. Lottici. Phys. Status Solidi B 154, K 117 (1989).

[10] V.V. Ursaki, I.I. Burlakov, I.M. Tiginyanu, Y.S. Raptis, E. Anastassakis, A. Aneda. Phys. Rev. B 59, 257 (1999).

[11] T. Mitani, S. Onari, K. Allakhverdiev, F. Gashimzade, T. Kerimova. Phys. Status Solidi B 223, 287 (2001).

[12] N.N. Syrbu, L.L. Nemerenco, O. Cojocaru. Cryst. Res. Technol. 37, 101 (2002).

[13] M. Fuentes-Cabrera. J. Phys.: Condens. Matter 13, 10117 (2001).

[14] Г.Г. Гусейнов, Т.Г. Керимова, Р.Х. Нани. Изв. АН ССР 4, 59 (1980).

[15] P. Gianozzi, S. de Gironcoli, P. Pavone, S. Baroni. Phys. Rev. B 43, 7231 (1991).

[16] S. Baroni, S. de Gironcoli, A. Dal Corso, P. Gianozzi. Rev. Mod. Phys. 73, 515 (2001).

[17] X. Gonze. Phys. Rev. B 55, 10337 (1997).

[18] X. Gonze, J.M. Beuken, R. Caracas, F. Detraux, M. Fuchs. G.M. Rignanese, L. Sindic, M. Verstraete, G. Zerah, F. Jallet. Comput. Mater. Sci. 25, 478 (2002).

[19] C. Hartwigsen, S. Goedecker, J. Hutter. Phys. Rev. B 58, 3641 (1998).

[20] J.P. Perdew, A. Zunger. Phys. Rev. B 23, 5048 (1981).

[21] H. Monkhorst, J. Pack. Phys. Rev. B 13, 5188 (1976).

[22] Р.К. Велиев, К.К. Мамедов, Г.Г. Гусейнов, Ф.М. Сеидов. Неорган. материалы 25, 1449 (1989).

[23] H. Hahn, G. Frank, W. Klinger, A.D. Storger, G. Storger, Z. Anorg. Allg. Chem. 279, 241 (1955).

[24] G.F. Mocharnyuk, T.I. Babyuk, L.S. Lazarenko, M.M. Markus, S.I. Radautsan. Book Fizicheskaya elektronika, Lvov, Вища шк., 15, 64 (1977).

[25] S.-H. Ma, Z.-Y. Jiao, X.-Z. Zhang. J. Mater. Sci. 47, 3849 (2012).

Редактор Д.В. Жуманов 